\title{
A characterization of quasi-rational polygons
}

\author{
Nicolas Bedaride*
}

\begin{abstract}
The aim of this paper is to study quasi-rational polygons related to the outer billiard. We compare different notions introduced in GS92 and [Sch09] and make a synthesis of those.
\end{abstract}

\section{Introduction}

The outer billiard map is a transformation $T$ of the exterior of a planar convex bounded domain $D$ defined as follows: $T(M)=N$ if the segment $M N$ is tangent to the boundary of $D$ at its midpoint, and $D$ lies at the right of $M N$. The outer billiard map is not defined if the tangent segment $M N$ shares more than one point with the boundary of $D$. In the case where $P$ is a convex polygon; the set of points for which $T$ or any of its iterations is not defined is contained in a countable union of lines and has zero measure. The dual billiard map has been introduced by Neumann in [Neu59] as a toy model for the planet orbits. One of the most interesting questions was whether the orbits of $T$ might escape to infinity for a polygonal domain $D$.

Two particular classes of polygons have been introduced by Kolodziej et al. in several articles, see Koł89, GS92, VS87. These classes are named rational and quasi-rational polygons and contain all the regular polygons. A rational polygon has vertices on a lattice of $\mathbb{R}^{2}$. They prove that every orbit outside a polygon in this class is bounded. Every regular polygon is a quasi-rational polygon, and it is not a rational polygon except if there are 3,4 or 6 edges. In the case of the regular pentagon, Tabachnikov completely described the dynamics of the outer billiard map in terms of symbolic dynamics, see [Tab95b]. He proves that some orbits are bounded and non periodic. The symbolic coding of this map has been given in [BC11 for a regular polygon with $3,4,5,6$ and 10 edges.

For non quasi-rational polygons, there is no general study. The case of trapezoids has been studied. The set of trapezoids can be parametrized up

\footnotetext{
${ }^{*}$ Laboratoire d'Analyse Topologie et Probabilités UMR 7353, Université Aix Marseille, avenue escadrille Normandie Niemen 13397 Marseille cedex 20, France. nicolas.bedaride@univ-amu.fr
} 
to affinity by one parameter. For an irrational parameter, it is not a quasirational polygon, and the proof of GS92 cannot be used for a polygon with parallel sides. Nevertheless, Li proved that all the orbits of the outer billiard map are bounded (this theorem is also proved by Genin) see [Li09] and Gen08. Recently Schwartz described a family of quadrilaterals, named kites, for which there exists unbounded orbits, see [Sch07] and [Sch09]. In these papers Schwartz introduces many tools in order to study the dynamics. These tools can also be used in the case of regular polygons, see [Sch10].

In this article we investigate the case of quasi-rational polygons. The main achievements of the paper consist of a synthesis of results of [GS92] and the notions introduced by Schwartz. These links allow us to give a new characterization of this class and to give some simple conditions which guarantee the quasi rationality.

Remark 1. In this article, $P$ is a polygon with $n$ vertices without parallel edges, see last section for some comments. All the figures correspond to the same polygon.

\section{Overview of the paper}

First we recall usual definitions about dual billiard in Section 3 and introduce our definition of quasi-rational polygon. Next, in Section 5, we show that our definition is equivalent to the old one of GS92 and also similar to Sch09. In Section 6 we prove the classical theorem on quasi-rational polygon using our definition. Finally in Section 8 we use our definition to obtain new results on quasi-rational polygons.

\section{Outer billiard}

We refer to Tab95a or GS92. We consider a convex polygon $P$ in $\mathbb{R}^{2}$ with $n$ vertices. Let $\bar{P}=\mathbb{R}^{2} \backslash P$ be the complement of $P$.

We fix an orientation on $\mathbb{R}^{2}$. We will define the outer billiard map off a countable union of lines. The map will be defined for all time.

For a point $M \in \bar{P}$, there are two half-lines $R, R^{\prime}$ emanating from $M$ and tangent to $P$, see Figure 1. Assume that the oriented angle $R, R^{\prime}$ has positive measure. Denote by $A^{+}, A^{-}$the tangent points on $R$ respectively $R^{\prime}$. We say that $A^{+}$is the vertex associated to $M$.

Definition 1. The outer billiard map is the map $T$ defined as follows:

$$
T(M)=r_{A^{+}}(M)
$$

where $r_{A^{+}}$is the reflection about $A^{+}$. 

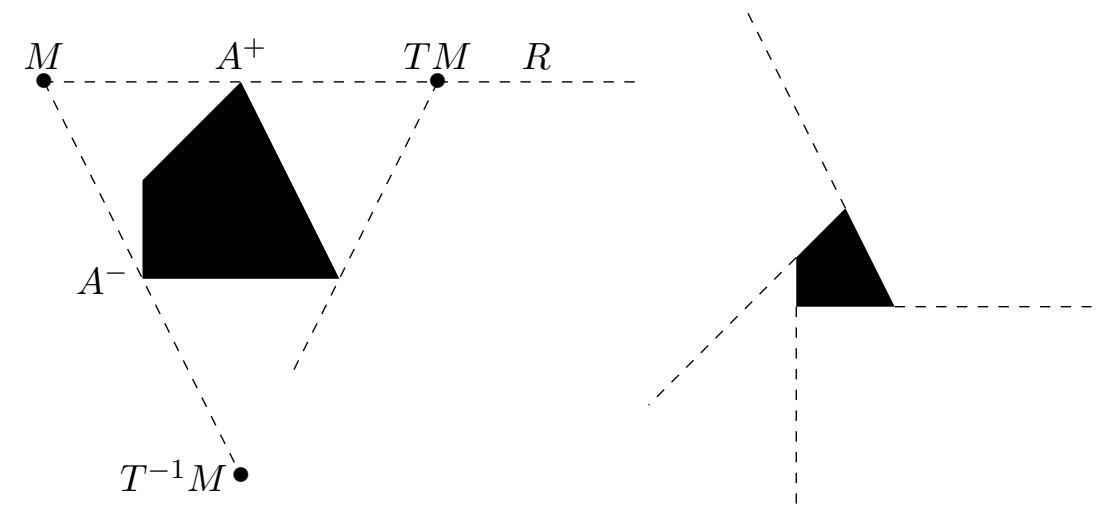

Figure 1: The outer billiard map

Definition 2. A polygon $P$ is said to be rational if the vertices of $P$ are on a lattice of $\mathbb{R}^{2}$.

We refer to Sch09. Consider a polygon without parallel edges. Assume the edges are oriented counterclockwise sense (while $T$ is oriented clockwise). For each edge, consider the vertex of $P$ furthest from the line supporting the edge. It is unique by convexity and assumption. Then denote by $V$ the vector equal to twice the vector between the final vertex of the edge and this vertex. A strip is the band formed by the line supporting the edge and $V$, see Figure 2, It is denoted $(\Sigma, V)$ or $\Sigma$ if no confusion is possible. We index them with respect to the slopes of the sides of the polygon, this gives the sequences $\left(\Sigma_{i}, V_{i}\right)_{1 \leq i \leq n}$.

Definition 3. Let $\alpha_{1}, \ldots, \alpha_{n}$ be non zero real numbers, we say that $\left(\alpha_{1}, \ldots, \alpha_{n}\right)$ are commensurate if $\frac{\alpha_{2}}{\alpha_{1}}, \ldots, \frac{\alpha_{n}}{\alpha_{n-1}}, \frac{\alpha_{1}}{\alpha_{n}}$ are rational numbers.

Definition 4. The polygon $P$ is quasi-rational if and only if $\left(\left|V_{1} \wedge V_{2}\right|, \ldots,\left|V_{n} \wedge V_{1}\right|\right)$ are commensurates.

For example, consider the polygon with vertices $A, B, C, D$, see Figure 2. The vectors are equal to: $V_{1}=2 \overrightarrow{C B}, V_{2}=2 \overrightarrow{A C}, V_{3}=2 \overrightarrow{B D}, V_{4}=2 \overrightarrow{B A}$.

\section{$4 \quad$ Unfolding}

In this Section we recall the notion of unfolding introduced in [GS92]. This notion is used to transform the outer billiard map in a piecewise translation map defined on cones.

\subsection{Definitions}

Consider two vertices $A, B$ of $P$, and the images of $P$ by the rotations $r_{A}, r_{B}$ of angle $\pi$. They are equal up to translation. Denote by $\tilde{P}$ one of 


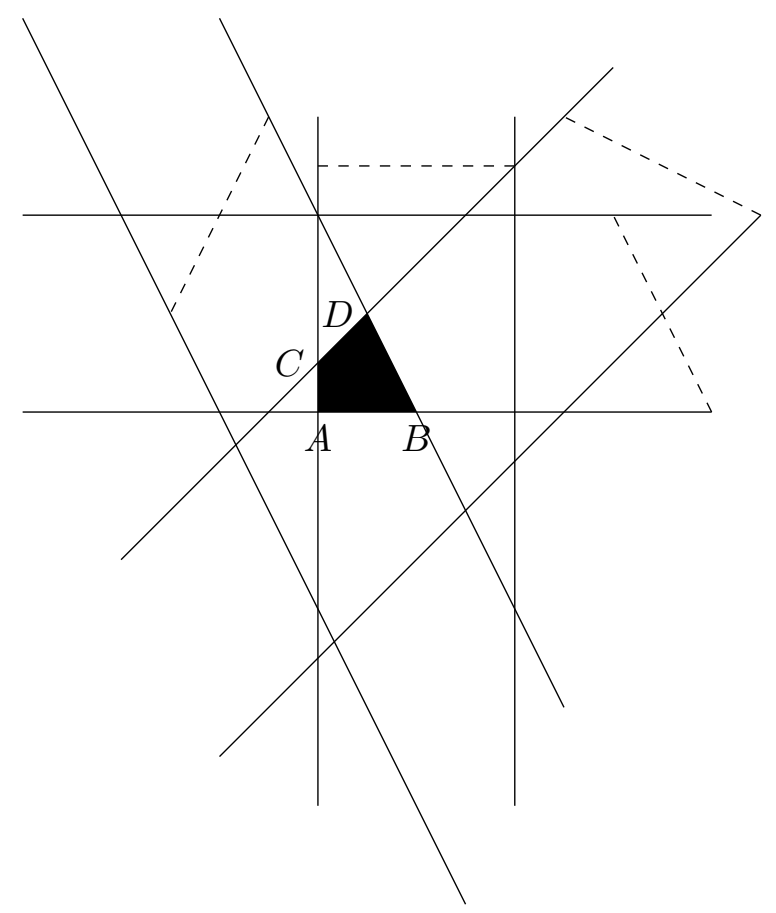

Figure 2: Polygon and strips

these polygons. Let $\mathcal{S}_{P}$ be the set of those polygons in $\mathbb{R}^{2}$ that are images by a translation by $P$ or $\tilde{P}$. Let $M$ be a point in $\bar{P}$, define the following bijective map.

$$
\begin{array}{rlcc}
\pi_{M}: \mathcal{S}_{P} & \rightarrow & \mathbb{R}^{2} \times\{-1,1\} \\
Q & \mapsto & (A, \varepsilon)
\end{array}
$$

Consider the image of $M$ by the outer billiard map outside $Q$. It is obtained by a rotation of angle $\pi$ centered at a vertex of $Q$. Let $A$ be this vertex of $Q$. Moreover we take $\varepsilon=1$ if $Q$ is a translate of $P, \varepsilon=-1$ if $Q$ is a translate of $\tilde{P}$. We say $A$ is associated to $M$ for $Q$. It is clear that $\pi_{M}$ is a bijection.

We define a new map called the unfolding of the dual billiard map.

$$
\begin{array}{ccc}
\tilde{T}: \mathbb{R}^{2} \times\{-1,1\} & \rightarrow & \mathbb{R}^{2} \times\{-1,1\} \\
(A, \varepsilon) & \mapsto & \left(A^{\prime},-\varepsilon\right)
\end{array}
$$

The ordered pair $(A, \varepsilon)$ comes from a polygon $Q$ via the map $\pi_{M}$. Consider the polygon $Q^{\prime}$ image of the polygon $Q$ by a rotation of angle $\pi$ of center $A$, see Figure 3. The point $A^{\prime}$ is the vertex associated to $M$ for $Q^{\prime}$.

The dynamics of this map is related to the outer billiard map by the following result. In what follows we will also denote by $\tilde{T}$ the projection of $\tilde{T}$ to $\mathbb{R}^{2}$. 


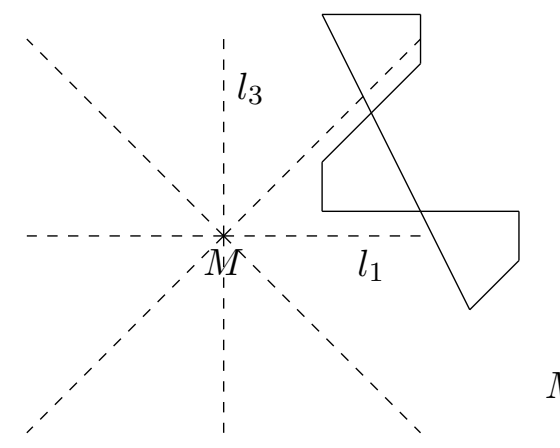

$M$

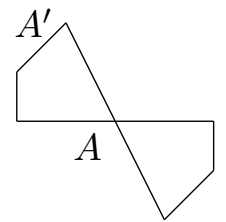

Figure 3: Necklace dynamics

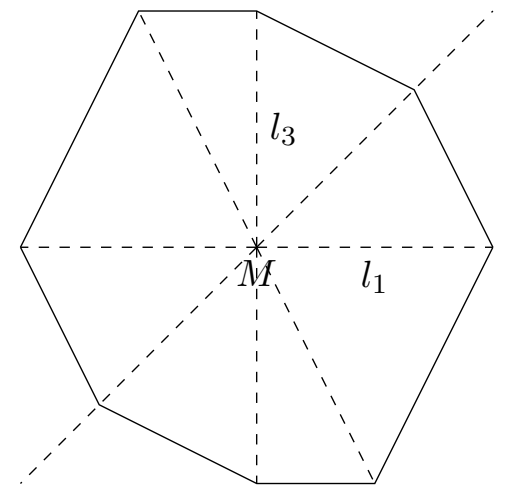

Figure 4: Polygon $P^{*}$ associated to the quadrilateral $A B C D$

Definition 5. Denote by $\left(l_{i}\right)_{i \leq n}$ the lines passing through $M$ and parallel to the edges of $P$. They defines $2 n$ cones $\left(C_{i}\right)_{1 \leq i \leq 2 n}$, each cone has for boundary two half lines $R_{i}, R_{i+1}$.

Proposition 1. GS92] We have:

- The sequence $\left(T^{k}(M)\right)_{k}$ is bounded (resp. periodic) if and only if there exists a point $Q \in \mathbb{R}^{2} \times\{-1,1\}$ such that the orbit of $Q$ is bounded (resp. periodic) for $\tilde{T}$.

- For every cone $C_{i}$, there exists a vector $a_{i}$ such that if $A, \tilde{T} A \in C_{i}$, then the restriction of $\tilde{T}$ to a cone is a translation of vector $a_{i}$. Moreover we have for every integer $i, a_{n+i}=-a_{i}$.

- There exists a polygon $P^{*}$ with $2 n$ edges, with vertices on $C_{1}, \ldots, C_{n}$ such that each side is parallel to some $a_{i}$.

The sides of $P^{*}$ will be denoted $v_{i}^{*}, i=1 \ldots 2 n$. 


\subsection{Some results}

Here we explain how to find the vectors $a_{1}, \ldots, a_{n}$.

Definition 6. For each cone $C_{i}$, let $d_{i}$ be a vector parallel to the edge $l_{i}$ such that $d_{i}+a_{i}$ is colinear to $l_{i+1}$.

Proposition 2. Consider the cone bounded by the lines $l_{i}, l_{i+1}$ and associated to the vector $a_{i}$. We have

- The strips associated to the lines $l_{i}$ and $l_{i+1}$ are consecutive for the slopes and $V_{i}=a_{i}$.

- The vectors $a_{i}, a_{i+1}$ have one vertex in common.

- The parallelogramm $\Sigma_{i} \cap \Sigma_{i+1}$ has $a_{i}$ for diagonal and $d_{i}$ for one side. The area of $\Sigma_{i} \cap \Sigma_{i+1}$ is equal to $\left|a_{i} \wedge d_{i}\right|$.

Proof. Consider the cone $C_{i}$ with boundaries $l_{i}, l_{i+1}$ and a polygon $Q \in \mathcal{S}_{P}$. Let $A$ be the vertex of $Q$ associated to $M$. The first thing to remark is that the slope of the line $(A M)$ is between the slopes of $l_{i}$ and $l_{i+1}$. Thus $A$ belongs to the edge parallel to $l_{i+1}$ and the point $\tilde{T} A$ belongs to the edge parallel to $l_{i}$. This proves $V_{i}=a_{i}$ and the first point.

Consider one strip with vertices $A, B, M$, it means that $M$ is the vertex that maximized the distance from $(A B)$. By definition the polygon is in the strip between $(A B)$ and $M+\mathbb{R} \overrightarrow{A B}$. Let $N$ be the vertex neighbour of $M$ in the polygon. Assume the vertex associated to $(M N)$ is not $B$, denote it $B^{\prime}$. The polygon is in the strip associated to $M, N, B^{\prime}$. Thus this strip does not intersect the segment $[A B]$. Then the line $(M N)$ has a slope bigger than $\left(B B^{\prime}\right)$. First part implies that in the ordering of the slopes, the slope of $(M N)$ is the consecutive of the slope of $(A B)$, contradiction.

The vector $d_{i}$ is on the boundary of $\Sigma_{i}$ by definition. Denote $a_{i}=v-w$ with $v, w$ vertices of $P$. By the previous point, there exists a vertex $w^{\prime}$ such that $w w^{\prime}$ is on the boundary of $\Sigma_{i+1}$. Thus one side of $\Sigma_{i} \cap \Sigma_{i+1}$ is given by the line $w w^{\prime}$ and one side by the line $d_{i}$. The area of the parallelogramm is the cross product of one side by the diagonal.

\subsection{Comments}

The preceding proposition may seem awkward, since we are not studying directly the outer billiard map to obtain results on its dynamics. Nevertheless we can transform the statement in terms of the outer billiard map $T$. The map $T^{2}$ is a piecewise translation, defined on several subsets of $\mathbb{R}^{2}$. Some of them can be compact sets, see Figure 5. Outside a compact region containing the polygon, the sets are unbounded and the translation vectors are two by two opposite. The translation vectors are exactly the vectors $V_{i}$, see Proposition 2. The dynamics of $T^{2}$ is simple: A point $m$ begins its 


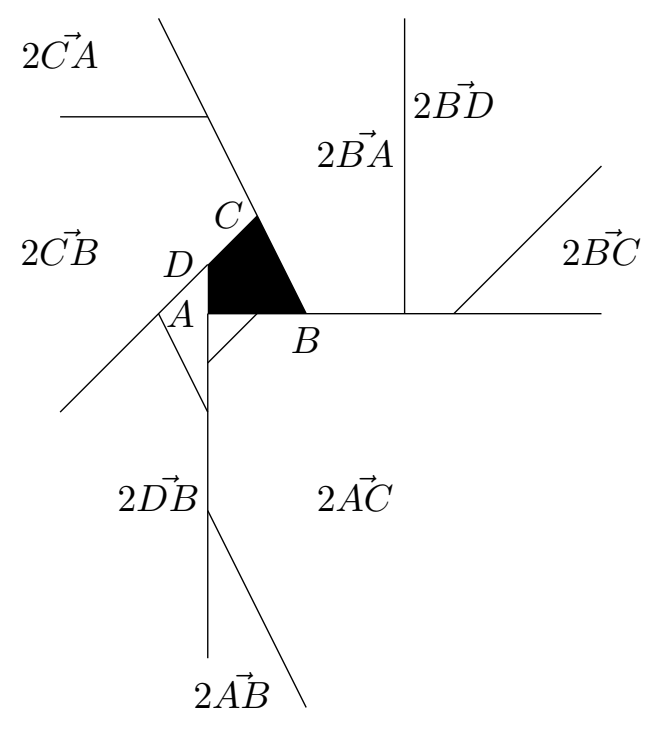

Figure 5: Definition of $T^{2}$

trajectory by being translated by a vector $V_{i}$ until it reaches another set where it moves by another vector $V_{i}$. Thus an orbit of point far away from $P$ looks like the polygon $P^{*}$. The link between $T^{2}$ and the piecewise translations of vectors $V_{1} \ldots V_{n}$ can be extended to a neighborhood of $P$, but it is much more complicated, see the Pinwheel theorem [Sch11]. It is related in Proposition 1 to the case where $A$ is closed to $M$. It is possible that the condition $A, \tilde{T} A \in C_{i}$ is not verified. This case is treated by the Pinwheel theorem in Sch09.

\section{Equivalence}

\subsection{Statement of results}

The aim is to prove

Theorem 1. The followings are equivalent:

- $\left(\frac{v_{1}^{*}}{\left|a_{1}\right|}, \ldots, \frac{v_{n}^{*}}{\left|a_{n}\right|}\right)$ are in $\mathbb{P Q}^{n}$.

- $\left(\left|a_{1} \wedge a_{2}\right|, \ldots,\left|a_{n} \wedge a_{1}\right|\right)$ are commensurates.

- $\left(\left|\Sigma_{2} \cap \Sigma_{1}\right|, \ldots,\left|\Sigma_{n} \cap \Sigma_{1}\right|\right)$ are commensurates.

Remark 2. The first point is the initial definition of a quasi-rational polygon given in [GS92]. The third is the definition by Schwartz in [Sch09]. 
To do this we will prove the three following propositions. The theorem will be a clear consequence with help of Proposition 2 .

Proposition 3. The followings are equivalent:

- There exists a rational solution $\left(t_{1}, \ldots, t_{n}\right)$ to

$$
\left\{\begin{array}{l}
d_{1}+a_{1}=t_{2} d_{2} \\
d_{1}+a_{1}+t_{2} a_{2}=t_{3} d_{3} \\
d_{1}+a_{1}+\ldots t_{n} a_{n}=-d_{1}
\end{array}\right.
$$

- $\left(\left|a_{1} \wedge d_{1}\right|, \ldots,\left|a_{n} \wedge d_{n}\right|\right)$ are commensurates.

Proposition 4. The followings are equivalent:

- There exists a rational solution $\left(t_{1}, \ldots, t_{n}\right)$ to

$$
\left\{\begin{array}{l}
d_{1}+a_{1}=t_{2} d_{2} \\
d_{1}+a_{1}+t_{2} a_{2}=t_{3} d_{3} \\
d_{1}+a_{1}+\ldots t_{n} a_{n}=-d_{1}
\end{array}\right.
$$

- $P$ is a quasi-rational polygon.

Proposition 5. The followings are equivalent:

- There exists a rational solution $\left(t_{1}, \ldots, t_{n}\right)$ to

$$
\left\{\begin{array}{l}
d_{1}+a_{1}=t_{2} d_{2} \\
d_{1}+a_{1}+t_{2} a_{2}=t_{3} d_{3} \\
d_{1}+a_{1}+\ldots t_{n} a_{n}=-d_{1}
\end{array}\right.
$$

- $\left(\frac{v_{1}^{*}}{\left|a_{1}\right|}, \ldots, \frac{v_{n}^{*}}{\left|a_{n}\right|}\right)$ are in $\mathbb{P} \mathbb{Q}^{n}$.

\subsection{Proof of Proposition 3}

The proof is based on Figure 6. Consider a polygon such that the points $A, B, C, D, E$ are vertices labelled in such a way that the slopes of edges are in the increasing order $A D, B C, B E$. Also assume we have $a_{1}=2 \overrightarrow{D C}$. Then Proposition 2 implies that $a_{2}=2 \overrightarrow{B D}$. Let $G$ be the intersection point of $(A D)$ and $(B C)$, and let $H$ a point on the line $(B C)$ such that $(H D)$ is parallel to $(B E)$. Then we have $d_{1}=2 \overrightarrow{G D}, d_{2}=2 \overrightarrow{H B}$. Moreover $\Sigma_{1} \cap \Sigma_{2}$ is defined by the triangle $G C D$, and $\Sigma_{3} \cap \Sigma_{2}$ is defined by $B D H$. Let $r$ be the real number such that $\overrightarrow{B H}=r \overrightarrow{G C}$. We see that $\Sigma_{1}$ is given by $((A D), 2 \overrightarrow{D C})$, $\Sigma_{2}=((B C), 2 \overrightarrow{B D})$. The intersection of the two strips $\Sigma_{1}, \Sigma_{2}$ has $D C$ as a 

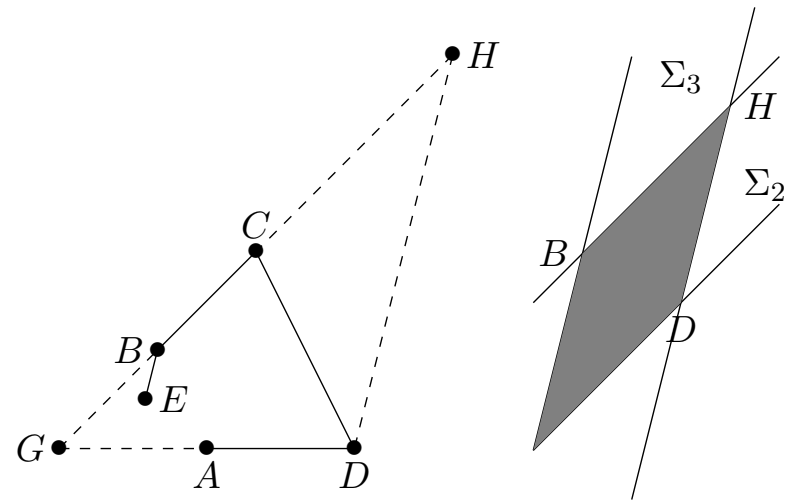

Figure 6: Proof of Proposition 3

diagonal. A similar computation gives the intersection of the strips $\Sigma_{3}, \Sigma_{2}$. The two parallelograms are constructed on triangles $B H D, G C D$. We have

$$
\begin{gathered}
|B H D|=|\overrightarrow{B H} \wedge \overrightarrow{B D}| \\
|G C D|=|\overrightarrow{G C} \wedge \overrightarrow{C D}|=|\overrightarrow{G C} \wedge \overrightarrow{B D}|=r|B H D| .
\end{gathered}
$$

The ratio of the areas of the two parallelograms is the same as the ratio of the area of triangles, thus it is equal to $r$. Thus we have proved:

$$
r \in \mathbb{Q} \Longleftrightarrow \frac{\left|\Sigma_{1} \cap \Sigma_{2}\right|}{\left|\Sigma_{2} \cap \Sigma_{3}\right|} \in \mathbb{Q} \text {. }
$$

Note that $r$ is equal to the inverse of $t_{2}$ in the first part of Proposition. The proof of Proposition follows by induction.

\subsection{Proof of Proposition 4}

The proof is based on Figure 7, Consider an edge $A D$, and the associated vector $a_{1}=2 \overrightarrow{D C}$. By Proposition 2 the vector $a_{2}$ is equal to $2 \overrightarrow{B D}$ with $B C$ edge of the polygon, and $a_{3}=2 \overrightarrow{F B}$, with $D F$ edge of the polygon. Let us call $G$ the intersection of $(C B)$ with $(A D)$, and $H$ the intersection of $(C B)$ and $(D F)$. Then $d_{1}=2 \overrightarrow{G D}, d_{2}=2 \overrightarrow{H B}$. Assume that the first item of Proposition 4 holds. Then there exist $r, r^{\prime} \in \mathbb{Q}$ such that $\overrightarrow{G C}=$ $r \overrightarrow{H B}, \overrightarrow{H D}=r^{\prime} \overrightarrow{D F}$. Solving system shows that $d_{i}$ is a rational linear sum of $a_{1}, \ldots, a_{n}$. By Proposition 2, the edges of $P$ are rational combination of $a_{1}, \ldots, a_{n}$, the assumption implies: $\overrightarrow{G C}=q \overrightarrow{B C}, \overrightarrow{H D}=q^{\prime} \overrightarrow{F D}$ with $q, q^{\prime} \in \mathbb{Q}$. Now the relations $\overrightarrow{G C}=q \overrightarrow{B C}=r \overrightarrow{H B}$ imply $\overrightarrow{H B}=q " \overrightarrow{C B}$ with $q^{\prime \prime}$ rational number. For a point $M$, denote $h_{M}$ the length of the orthogonal projection of $M$ on $(D B)$. The relations $\overrightarrow{H B}=q " \overrightarrow{C B}, \overrightarrow{H D}=q \overrightarrow{F D}$ gives $h_{H}=q " h_{C}$ and $h_{F}=q h_{H}$. By Proposition 2, the areas $\left|a_{1} \wedge a_{2}\right|,\left|a_{2} \wedge a_{3}\right|$ are given by 
areas of triangles $B C D, D B F$. The ratio of these areas is equal to the ratio between $h_{C}$ and $h_{F}$. Thus the areas are commensurates. The other part of the proof is similar.

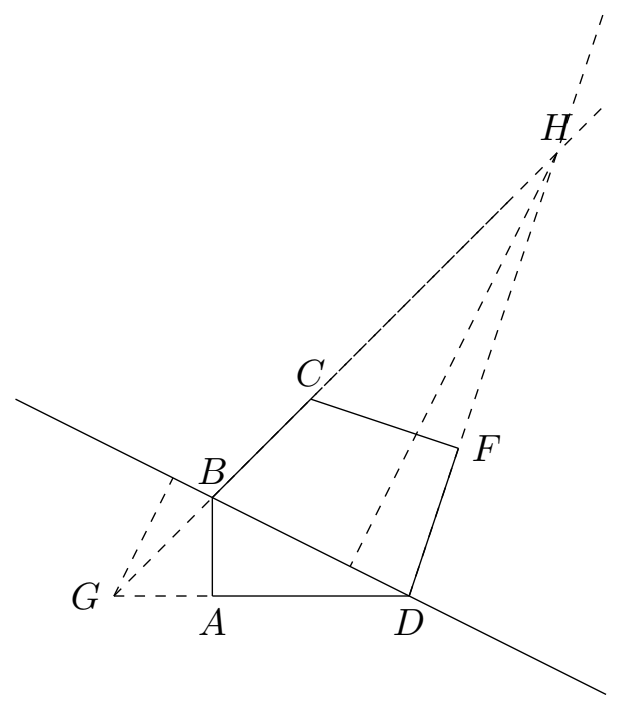

Figure 7: Proof of Proposition 4

\subsection{Proof of Proposition 5}

The proof is based on Figure 4. By Proposition 4 we know that the first statement is equivalent to the fact that $P$ is a quasi-rational polygon. If the system has a rational solution, then the polygon defined by $d_{1}, d_{1}+$ $a_{1}, \ldots, d_{1}+a_{1}+\ldots t_{n} a_{n}$ is some polygon $P^{*}$. The edges $v_{1}^{*}, \ldots, v_{n}^{*}$ of this polygon are equal to $a_{1}, t_{2} a_{2}, \ldots, t_{n} a_{n}$, thus the first implication is proved.

Conversly, consider the point $M$ on $l_{1}$ such that $O \vec{M}=d_{1}$. By hypothesis, there exists a polygon with sides $r_{1} a_{1}, \ldots r_{n} a_{n}$ with rational numbers $r_{i}, i \leq n$. Thus there exists an homothetic image of this polygon with vertex $M$, and all the edges fulfilling the same condition. This gives a rational solution of the system.

This proposition can be reformulated in

Corollary 1. The polygon is quasi-rational if and only if : there exists 
rational numbers $t_{2}, \ldots, t_{n}$ and $M \in \mathbb{R}^{2}$ such that

$$
\left\{\begin{array}{l}
M \in l_{1} \\
M+a_{1} \in l_{2} \\
M+a_{1}+t_{2} a_{2} \in l_{2} \\
M+a_{1}+\cdots+t_{i} a_{i} \in l_{i} \\
M+a_{1}+\cdots+t_{n} a_{n}=-M
\end{array}\right.
$$

\section{All orbits are bounded for quasi-rational poly- gons}

In this section we give a new proof of the following theorem using our definition of quasi-rational polygon. The aim is to understand the general outline of the proof, not to explain all the details.

Theorem 2. [GS92] For a quasi-rational polygon, every orbit of the dual billiard map is bounded.

We consider the unfolding and the cone $C_{1}$. We can tile periodically this cone by a parallelogramm with one side equal to $d_{1}$ and one diagonal equal to $a_{1}$. The same thing can be done in all cones. Consider a point $x$ and the first hitting map with the next cone: $f_{1}$. We have $f_{1}\left(x+d_{1}\right)=f_{1}(x)+a_{1}+d_{1}$, we deduce $f_{2}\left(f_{1}\left(x+d_{1}\right)\right)=f_{2}\left(f_{1}(x)+t_{2} d_{2}\right)$. Since the polygon is quasirational there exists an integer $n$ such that $f_{2}\left(f_{1}\left(x+n d_{1}\right)\right)=f_{2}\left(f_{1}(x)+\right.$ $\left.n t_{2} d_{2}\right)=f_{2}(x)+n^{\prime}\left(a_{2}+d_{2}\right)$. Now the first return map to the cone $C_{1}$ is the composition of $f_{1}, \ldots, f_{n}$. We obtain that there exists a vector $u$ such that for every $x$

$$
F(x+u)=F(x)+u
$$

In term of parallelograms, it means that we consider a point in one box and take the image of the box by $F$. We have a second periodic tiling of the cone by a parallelogram with side $u$ and diagonal $a_{1}$. The orbit of the point $x$ depends on the cutting of a box of the new tiling by the initial one. If the two tilings are commensurates then every orbit is bounded. We must compare $u$ and $d_{1}$ : they are rationally proportional by definition of quasirational polygon. If $P$ is rational every box is mapped by $F$ to a box, thus every orbit is periodic.

\section{$7 \quad$ Graph of spokes}

\subsection{Definitions}

By definition, for each integer $i, a_{i}$ is a vector between two vertices of $P$, and every vertex is a starting point of some $a_{i}$. Define a graph with vertices the 


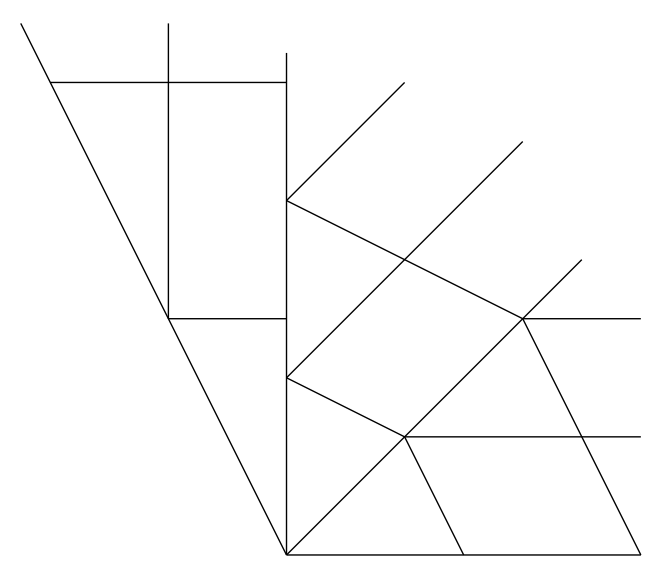

Figure 8: Tilings of consecutive cones

vertices of $P$, and there is an oriented edge starting from each vertex and joining the end of the vector $a_{i}$ associated to the vertex. Denote it $\mathcal{S}(P)$, and we call it the graph of spokes.

Example 1. Consider the polygon $A B C D$ of Figure 1 , then $\mathcal{S}(P)$ is given by

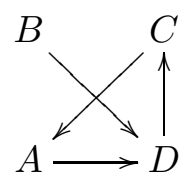

Figure 9: Graph of spokes

Lemma 1. This graph has following properties:

- Each vertex has an outgoing edge.

- Two edges can not have the same vertices.

- The graph contains a cycle.

Proof. Proof left to the reader for the two first items. An edge between vertices $A, B$ implies that some vector $a_{i}=\overrightarrow{A B}$. Thus the graph is the same thing as a map defined on the set of vertices. This map is defined everywhere but not necessarily injective. It is injective on a subset. On this subset the graph is a cycle.

Corollary 2. For every polygon, there exists a rational relation between the vectors $a_{1}, \ldots, a_{n}$. 
Proof. By preceding Lemma there exists a cycle in the graph. It implies that the sum of vectors $a_{i}$ associated to this cycle is null.

Remark 3. The notion of spokes is used in outer billiard by Schwartz to prove its result on the pinwheel map, see [Sch10].

We now use the preceding tools to obtain new results on quasi-rational polygons.

\section{Description of quasi-rational polygons}

Theorem 3. We have:

- A quadrilateral is a quasi-rational polygon if and only if it is rational.

- There exists a non regular and non rational quasi-rational pentagon.

- Assume the graph of spokes is a cycle (or an union of cycles). Then the polygon is quasi-rational.

Proof. - Consider $\left(a_{1}, a_{2}\right)$ as a basis of $\mathbb{R}^{2}$, denote $\alpha, \beta$ the coordinates of $a_{3}$ in this basis, and $(\gamma, \delta)$ those of $a_{4}: a_{3}=\alpha a_{1}+\beta a_{2}, a_{4}=\gamma a_{1}+\delta a_{2}$. The numbers $\left|a_{1} \wedge a_{2}\right|,\left|a_{3} \wedge a_{2}\right|,\left|a_{4} \wedge a_{3}\right|,\left|a_{4} \wedge a_{1}\right|$ are proportional to $1, \alpha, \alpha \delta-\beta \gamma, \delta$. If the polygon is quasi-rational, by Theorem 1 we deduce $\alpha, \delta, \alpha \delta-\beta \gamma \in \mathbb{Q}$. This implies $\beta \gamma \in \mathbb{Q}$. Now Corollary 2 implies that there exists a rational linear relation between $a_{1}, \ldots, a_{4}$. This relation concerns at least three vectors. All possibilities imply $\beta, \gamma \in \mathbb{Q}$. Thus the polygon has vertices on a lattice.

- Consider four points on a lattice of $\mathbb{R}^{2}$. Denote these points $A, B, C, E$. We will contruct a point $D$ such that the pentagon $A B C D E$ will be as required. It suffices to consider one point $D$ outside the lattice. We can always choose $D$ such that the spokes of the pentagon $A B C D E$ are associated to vectors $\overrightarrow{A C}, \overrightarrow{B E}, \overrightarrow{C E}, \overrightarrow{D B}, \overrightarrow{E A}$. Then the rational relation is $a_{1}+a_{3}+a_{5}=0$. There is no other rational relation by definition of $D$. Now we can express the vectors $a_{1}, \ldots, a_{5}$ in the basis $\left(a_{1}, a_{2}\right)$. By construction $a_{3}, a_{5}$ have rational coordinates. Then we can always choose $D$ such that the area $\left|a_{2} \wedge a_{3}\right|$ is rational. The constructed pentagon is quasi-rational.

- Now assume that the graph of spokes is an union of cycles. By Corollary 1 a polygon is quasi-rational if for every side $l_{i}$, there exists $\lambda \in \mathbb{R}$ and rational numbers $r_{1}, \ldots, r_{n} \in \mathbb{Q}^{*}$ such that

$$
\lambda l_{i}+r_{1} a_{1}+\cdots+r_{n} a_{n}=0 .
$$


If the graph is a union of cycles, then the map defined on vertices associated to the graph of spokes is invertible. It means that each vertex is a linear combination of $a_{1} \ldots a_{n}$. Thus the side $l_{i}$ can be expressed as rational combination of the $a_{i}$ 's. Thus $P$ is quasi-rational if there exists $r_{1} \ldots r_{n} \in \mathbb{Q}$ and $\lambda \in \mathbb{R}$ such that:

$$
\lambda \sum r_{j}^{\prime} a_{j}+r_{1} a_{1}+\cdots+r_{n} a_{n}=0 .
$$

Since the graph is a cycle, there exists a rational relation between $a_{1} \ldots a_{n}$. Thus we can solve the equation and find $r_{1} \ldots r_{n}, r_{1}^{\prime} \ldots r_{n}^{\prime}$.

Remark 4. Consider the example of graph in Figure 9. In this case the preceding map is not a bijection since no edge goes to $B$.

For regular polygon with odd number of sides (greater than five), the graph is not simply connected.

\section{Remarks}

\subsection{Polygon with parallel sides}

If the polygon has parallel sides, then the definition of [GS92] still works. Nevertheless the number of cones decreases. For the definition of [Sch09] we need to be more precise to define a strip. In this case two consecutive strips can have an intersection with infinite area. Thus the new definition of quasi-rational is that, up to a factor, the areas of $\Sigma_{i} \cap \Sigma_{i+1}$ are in $\mathbb{Z} \cup\{\infty\}$ for every integer $i$.

\subsection{Regular polygons}

A regular polygon with $n$ edges is invariant by rotation of angle $2 \pi / n$. Let $\omega$ be a $n$th root of unity, we have $a_{i}=\omega a_{i-1}+a_{i-2}$ for every integer $i$. Thus it is clear that $\left|a_{i} \wedge a_{i+1}\right|$ is a constant number, and a regular polygon is a quasi-rational polygon. Moreover the graph of spokes is a cycle, since the spoke $a_{i+1}$ is the image of $a_{i}$ by rotation of angle $2 \pi / n$. This gives another proof of previous fact.

The study of regular polygons has been done if the number of sides is equal to 5 by Tabachnikov, see Tab95b. A description of the symbolic dynamics has been made for regular polygons with 3,4,5,6,10 edges in [BC11]. In Sch10] Schwartz initiates a study of the regular octogon. 


\section{References}

[BC11] N. Bedaride and J. Cassaigne. Outer billiards outside regular polygons. Journal of London Mathematical Society, 2(83):301-323, 2011.

[Gen08] D. Genin. Research announcement: boundedness of orbits for trapezoidal outer billiards. Electron. Res. Announc. Math. Sci., 15:71-78, 2008.

[GS92] E. Gutkin and N. Simányi. Dual polygonal billiards and necklace dynamics. Comm. Math. Phys., 143(3):431-449, 1992.

[Koł89] R. Kołodziej. The antibilliard outside a polygon. Bull. Polish Acad. Sci. Math., 37(1-6):163-168 (1990), 1989.

[Li09] L. Li. On Moser's boundedness problem of dual billiards. Ergodic Theory Dynam. Systems, 29(2):613-635, 2009.

[Neu59] B.H.R. Neumann. Sharing ham and eggs. Iota, Manchester university Mathematics students journal, 1959.

[Sch07] R. E. Schwartz. Unbounded orbits for outer billiards. I. J. Mod. Dyn., 1(3):371-424, 2007.

[Sch09] R. E. Schwartz. Outer billiards on kites, volume 171 of Annals of Mathematics Studies. Princeton University Press, Princeton, NJ, 2009.

[Sch10] R. E. Schwartz. Outer billiards, the arithmetic graph and the octagon. Arxiv, 2010.

[Sch11] Richard Evan Schwartz. Outer billiards and the pinwheel map. J. Mod. Dyn., 5(2):255-283, 2011.

[Tab95a] S. Tabachnikov. Billiards. Panoramas et Synthèses, 1995.

[Tab95b] S. Tabachnikov. On the dual billiard problem. Adv. Math., 115(2):221-249, 1995.

[VS87] F. Vivaldi and A. V. Shaidenko. Global stability of a class of discontinuous dual billiards. Comm. Math. Phys., 110(4):625-640, 1987. 